\title{
Generation of reactive oxygen species by the faecal matrix
}

\author{
R W Owen, B Spiegelhalder, H Bartsch
}

\begin{abstract}
Background-Reactive oxygen species are implicated in the aetiology of a range of human diseases and there is increasing interest in their role in the development of cancer.

Aim-To develop a suitable method for the detection of reactive oxygen species produced by the faecal matrix.

Methods-A refined high performance liquid chromatography system for the detection of reactive oxygen species is described.

Results-The method allows baseline separation of the products of hydroxyl radical attack on salicylic acid in the hypoxanthine/ xanthine oxidase system, namely 2,5-dihydroxybenzoic acid, 2,3-dihydroxybenzoic acid, and catechol. The increased efficiency and precision of the method has allowed a detailed evaluation of the dynamics of reactive oxygen species generation in the faecal matrix. The data show that the faecal matrix is capable of generating reactive oxygen species in abundance. This ability cannot be attributed to the bacteria present, but rather to a soluble component within the matrix. As yet, the nature of this soluble factor is not entirely clear but is likely to be a reducing agent.

Conclusions-The soluble nature of the promoting factor renders it amenable to absorption, and circumstances may exist in which either it comes into contact with either free or chelated iron in the colonocyte, leading to direct attack on cellular DNA, or else it initiates lipid peroxidation processes whereby membrane polyunsaturated fatty acids are attacked by reactive oxygen species propagating chain reactions leading to the generation of promutagenic lesions such as etheno based DNA adducts.

(Gut 2000;46:225-232)
\end{abstract}

Keywords: colorectal cancer; faecal matrix; hypoxanthine; phytic acid; reactive oxygen species; xanthine oxidase

Division of Toxicology and Cancer Risk Factors, German Cancer Research Center, Im

Neuenheimer Feld 280, D-69120 Heidelberg,

Germany

R W Owen

B Spiegelhalder

H Bartsch

Correspondence to:

Dr R W Owen.

Accepted for publication

9 September 1999 itself or else through lipid peroxidation, a reaction initiated by hydrogen abstraction from polyunsaturated fatty acids by $\mathrm{HO}^{*}$ leading to a chain of events, resulting in the formation of 4-hydroxynonenal, which after epoxidation can lead to production of promutagenic DNA adducts - for example, etheno bridged adducts - and if misrepaired may be an early event in carcinogenesis. ${ }^{10}$

That free radicals generated within the intestinal lumen may have a role to play in colorectal carcinogenesis was first brought to our attention by the data of Graf and Eaton, ${ }^{11}$ who suggested that the protective effects of dietary fibre ${ }^{12}$ were in part mediated by the hexaphosphorylated sugar phytic acid, and this hypothesis has received support from several experimental ${ }^{13-15}$ and animal ${ }^{16-19}$ studies. The ameliorative mechanism is thought to function by chelation of iron by phytic acid in the large bowel preventing its involvement in Fenton chemistry and thereby the generation of reactive oxygen species, a contention that has also received support in animal model studies ${ }^{20-22}$ on the interactive effects of phytic acid and iron in relation to colorectal cancer. The strong interaction between phytic acid and iron in the faecal matrix has been confirmed by Owen et al. ${ }^{23}$

To date there is not a great deal of direct evidence implicating the above mechanism in the initiation of colorectal cancer in humans, the data of Simmonds and colleagues ${ }^{4}$ and Keshevarzian and colleagues ${ }^{78}$ apart, but it is possible that the faecal stream may exacerbate the process, either through the indigenous bacteria or by an as yet unknown component within the intestinal lumen.

The only reports of the faecal matrix being involved in the generation of reactive oxygen species are those of $\mathrm{Babbs}^{24}$ and Erhardt et $a l^{25}$ who put forward the hypothesis that this phenomenon may be important in the genesis of colorectal cancer, and Owen and colleagues $^{26}{ }^{27}$ Babbs $^{24}$ showed using dimethyl sulphoxide as a probe that 1:100 dilutions of rat faeces generated abundant amounts of reactive oxygen species $(1700 \mathrm{nmol} / \mathrm{g})$ and concluded that, because autoclaved faeces were ineffective, this was the end product of bacterial metabolism. Erhardt and colleagues ${ }^{25}$ showed that the generation of $\mathrm{HO}^{\circ}$ by human faeces was 13 times greater in subjects consuming a high fat/low fibre diet than in those consuming a low fat/high fibre diet. These were

Abbreviations used in this paper: EDTA, ethylenediaminetetraacetic acid; HPLC, high performance liquid chromatography; $\mathrm{HO}$, hydroxyl radical; 2,5-DHBA, 2,5-dihydroxybenzoic acid; 2,3-DHBA, 2,3-dihydroxybenzoic acid; $\mathrm{IC}_{50}$, concentration causing $50 \%$ inhibition. 


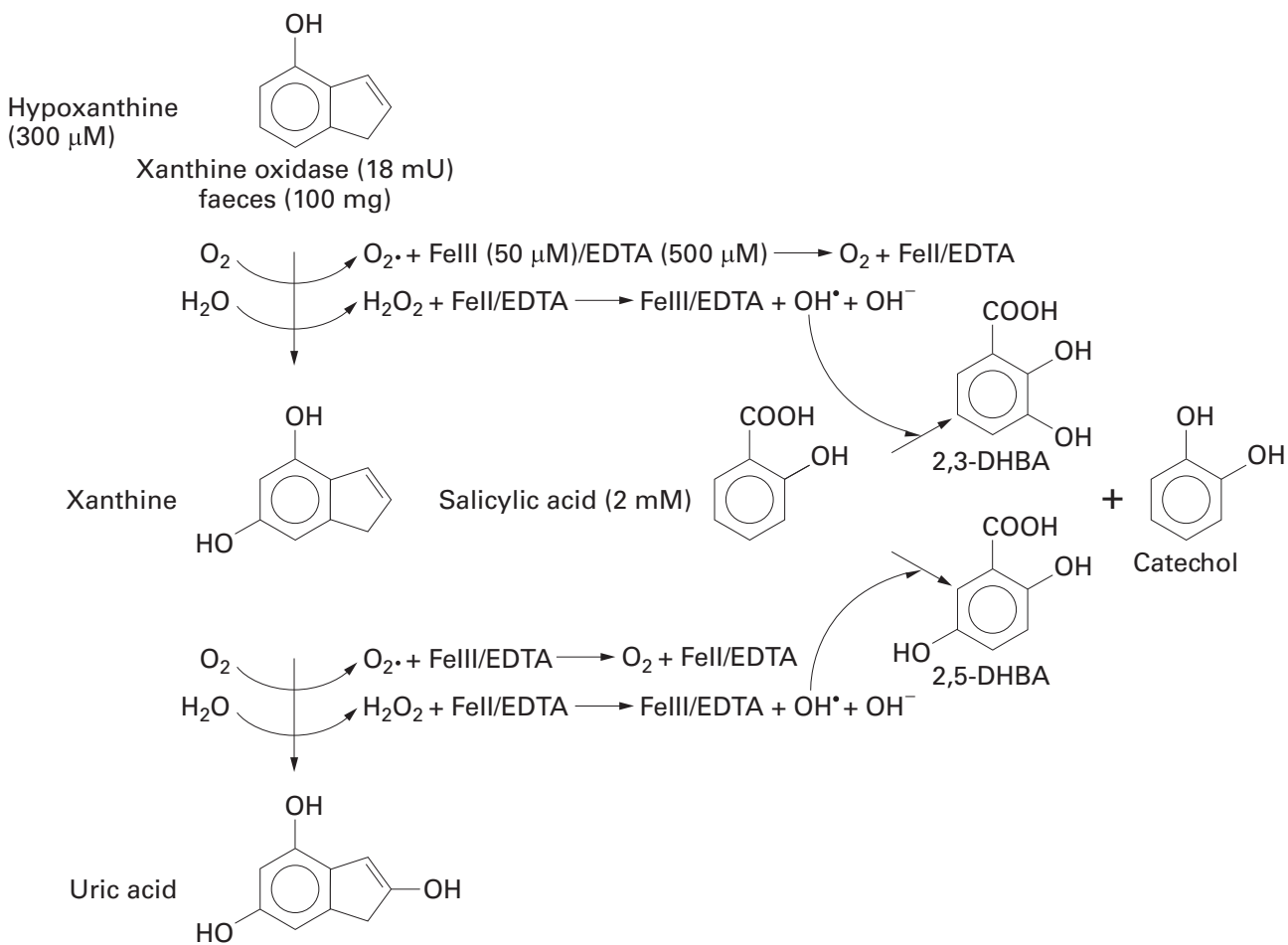

Figure 1 Reaction sequence for the analysis of reactive oxygen species in the hypoxanthine/xanthine oxidase and faecal systems.

very interesting discoveries, but the method $^{28}$ used to detect reactive oxygen species - that is, production of methylsulphinic acid from dimethyl sulphoxide and reaction of this with fast blue and subsequent colorimetric determination, is rather laborious. Furthermore, although the method works reasonably well in a standardised in vitro system, in our hands this was not the case when working with the faecal matrix. To overcome these problems, we developed ${ }^{26}{ }^{27}$ a high performance liquid chromatography (HPLC) method for the detection of reactive oxygen species in biological matrices, and our data for human faeces support some of the observations of Babbs. ${ }^{24}$ In this communication we describe a refined HPLC method which has enabled a detailed analysis of the dynamics of the system within the faecal matrix.

\section{Materials and methods}

REAGENTS

Ascorbic acid, ATP, xanthine, hypoxanthine, xanthine oxidase, ethanol, dimethyl sulphoxide, Tris and ethylenediaminetetraacetic acid (EDTA) were obtained from Merck (Darmstadt, Germany). Brain heart infusion broth, $\mathrm{K}_{2} \mathrm{HPO}_{4}$, and $\mathrm{KH}_{2} \mathrm{PO}_{4}$ were obtained from Serva (Heidelberg, Germany). Catechol, uric acid, salicylic acid, 2,5-dihydroxybenzoic acid (2,5-DHBA), 2,3-dihydroxybenzoic acid (2,3DHBA), $\alpha$-D-glucose, phytic acid $(40 \%$ in water), butanol, and $\mathrm{FeCl}_{3} \cdot 6 \mathrm{H}_{2} \mathrm{O}$ were obtained from Aldrich Chemie (Steinheim, Germany). Catalase (EC 1.11.1.6), bilirubin, hae$\mathrm{min}$, and biliverdin were obtained from Sigma Chemie (Deisenhofen, Germany). Sterile pyrogen-free disposable filter $(0.2 \mu \mathrm{m})$ holders were obtained from Schleicher and Schuell (Dassel, Germany). All solutions were made up in Millipore grade water.
FAECAL SAMPLES

Faecal samples were drawn from a long term placebo controlled calcium intervention study as described elsewhere. ${ }^{29}$ Samples were defecated into plastic bowls and placed immediately on solid carbon dioxide before transportation to the laboratory. They were stored at $-80^{\circ} \mathrm{C}$ until analysis. Samples were thawed overnight at $4^{\circ} \mathrm{C}$, pooled, and homogenised (room temperature) at $600 \mathrm{rpm}$ for five minutes in a rotating homogeniser. Aliquots of $40 \mathrm{~g}$ were freeze-dried, reweighed, and ground to a fine homogeneous powder with a mortar and pestle before analysis. The freeze-dried and remaining wet aliquots were refrozen at $-80^{\circ} \mathrm{C}$.

\section{HYPOXANTHINE/XANTHINE OXIDASE MODEL HO} GENERATING SYSTEM

This was constructed as depicted in fig 1. A detailed description is given in Owen et al. ${ }^{26} 27$ In the current studies, Tris $(50 \mathrm{mM})$ and phosphate $(100 \mathrm{mM})$ buffers were compared, because it has been reported that Tris is an efficient scavenger of reactive oxygen species ${ }^{30}$ and attenuates the signals produced during HPLC by HO' attack on salicylic acid. For pH studies, Tris buffer was altered as required by the addition of concentrated $\mathrm{HCl}$, and phosphate buffers were made up according to standard procedures. The effect of various constituents in the buffer systems on generation of reactive oxygen species was studied by either addition or omission, but for scavenger and enzyme inhibition studies the requisite amount of complete buffer system was replaced by a relevant volume of test material. Experiments were conducted in duplicate. 
FAECAL GENERATION OF REACTIVE OXYGEN SPECIES In a previous study, ${ }^{27}$ a basic screen of faecal samples (58) drawn from a long term placebo controlled calcium intervention trial as described elsewhere ${ }^{29}$ to support Fenton chemistry was conducted. The assays were conducted in Tris buffer, which, as already mentioned, is unlikely to give a true reflection of their ability to generate reactive oxygen species. In the current study, analyses were conducted in phosphate buffer, and four faecal specimens were studied in detail. The basal medium, either Tris (50 $\mathrm{mM}$ ) or phosphate buffer $(100 \mathrm{mM})$, contained EDTA $(500 \mu \mathrm{M}), \mathrm{FeCl}_{3} \cdot 6 \mathrm{H}_{2} \mathrm{O}(50 \mu \mathrm{M}$ with respect to elemental iron), and salicylic acid $(2 \mathrm{mM})$ suspended in Millipore grade water, final $\mathrm{pH}$ 6.5. Duplicate Erhlenmeyer flasks $(100 \mathrm{ml})$ containing $10 \mathrm{ml}$ medium, inoculated with $100 \mathrm{mg}$ faeces (wet, filter sterilised after thorough homogenisation in the buffer for one hour at $4^{\circ} \mathrm{C}$, or freeze-dried (1:100 dilution)) were incubated at $37^{\circ} \mathrm{C}$ in an orbital shaker (Grant Instruments, Cambridge, UK) at 200 strokes/min for 18 hours. After incubation followed by centrifugation and filtration, the cultures $(20 \mu \mathrm{l})$ were injected directly on to the HPLC column or else acidified with $50 \mu \mathrm{l}$ concentrated $\mathrm{HCl}$, extracted with $2 \times 10 \mathrm{ml}$ diethyl ether, centrifuged (5000 rpm for 30 minutes), and the solvent removed by aspiration. Ethereal extracts were dried over anhydrous $\mathrm{MgSO}_{4}$, the solvent was removed under a stream of $\mathrm{N}_{2}$, and the extracts were resuspended in $2 \mathrm{ml}$ mobile phase. After filtration, they $(20 \mu \mathrm{l})$ were analysed by HPLC.

GENERATION OF REACTIVE OXYGEN SPECIES BY BACTERIA

To elucidate whether or not microorganisms within the faecal samples were responsible for the generation of reactive oxygen species, the indigenous bacteria were cultured in brain heart infusion broth. For the culture of aerobic organisms the medium $(100 \mathrm{ml}$ in $250 \mathrm{ml}$ Erhlenmeyer flasks) was inoculated with $100 \mathrm{mg}$ wet faeces and incubated at $37^{\circ} \mathrm{C}$ for 18 hours on a shaking water bath (Grant Instruments) at 200 strokes/min. For the culture of anaerobic organisms, the medium $(100 \mathrm{ml}$ in $100 \mathrm{ml}$ Duran bottle) was supplemented with reducing agents (30 mg/l sodium formaldehyde sulphoxylate and $50 \mathrm{mg} / \mathrm{l} \mathrm{L}$-cysteine hydrochloride) and preincubated in an anaerobic churn in an atmosphere of hydrogen $(90 \%)$ and $\mathrm{CO}_{2}(10 \%)$ before inoculation with faeces $(1 \mathrm{~g})$ and re-incubation at $37^{\circ} \mathrm{C}$ for 48 hours. After incubation, the cultures were centrifuged at $5000 \mathrm{rpm}$ for 30 minutes; the cell pellets were harvested, washed twice in fresh phosphate buffer (containing reducing agents for the anaerobic organisms), and used immediately for the assay of reactive oxygen species. The cell pellets were serially diluted in the range $10^{1}-10^{3}$ directly in the faecal assay buffer and incubated at $37^{\circ} \mathrm{C}$ for 18 hours. After incubation, aliquots of the assay mixtures were either, after filter sterilisation, injected $(20 \mu \mathrm{l})$ directly on to the HPLC column or, after acidification, extracted twice with diethyl ether and concentrated before analysis as described for the faecal samples.
TIME COURSE STUDIES

Time course studies were conducted directly by HPLC at room temperature. For the hypoxanthine/xanthine oxidase system, complete phosphate buffer $(2.0 \mathrm{ml})$ was added to an HPLC vial and the reaction started by addition of xanthine oxidase. Faecal samples (100 mg wet weight) were thoroughly homogenised and filter sterilised before HPLC. The HPLC system was programmed to inject every 30 minutes, with a 10 minute reconditioning period. The approximate time point between each assay run was 40 minutes.

HIGH PERFORMANCE LIQUID CHROMATOGRAPHY Analytical HPLC was conducted on a HewlettPackard HP 1090 liquid chromatograph fitted with a C-18 (Latex, Eppelheim, Germany) reverse phase $25 \mathrm{~cm}(5 \mu \mathrm{m})$ column (internal diameter, $4.5 \mathrm{~mm}$ ). Salicylic acid and its dihydroxy derivatives were detected with a UV photodiode spectrophotometer set at $325 \mathrm{~nm}$ and for catechol at $278 \mathrm{~nm}$ at room temperature. The amount of diphenol produced was calculated from standard curves of both 2,5-DHBA and 2,3-DHBA constructed from chromatograms run at $325 \mathrm{~nm}$ in the range $0-1$ $\mathrm{mM}$. A similar standard curve was generated for catechol at $278 \mathrm{~nm}$.

For the separation of individual compounds, the mobile phase consisted of $2 \%$ acetic acid in water (solvent A) and methanol (solvent B) using the following gradient over a total run time of 45 minutes: $95 \% \mathrm{~A} / 5 \% \mathrm{~B}$ for two minutes, $75 \% \mathrm{~A} / 25 \% \mathrm{~B}$ for eight minutes, $60 \% \mathrm{~A} / 40 \% \mathrm{~B}$ for 10 minutes, $50 \% \mathrm{~A} / 50 \% \mathrm{~B}$ for 10 minutes, and $0 \% \mathrm{~A} / 100 \% \mathrm{~B}$ until completion of the run.

Incubates were either (after filtration) injected directly or, after acidification and extraction, dissolved in mobile phase $(2 \mathrm{ml})$ before injection $(20 \mu \mathrm{l})$ on to the HPLC column. The optimal flow rate of the mobile phase was $1 \mathrm{ml} / \mathrm{min}$. Instrument control and data handling were performed with an HP Chemstation operating in the Microsoft Windows software environment. Concentrations causing 50\% inhibition $\left(\mathrm{IC}_{50}\right.$ ) were calculated using Tablecurve (Jandel Scientific, Chicago, Illinois, USA).

\section{Results}

HIGH PERFORMANCE LIQUID CHROMATOGRAPHY In a previous communication, ${ }^{26}$ we described the detection of dihydroxybenzoic acids by an HPLC method based on ion-pair chromatography. The separation of the two major products of $\mathrm{HO}^{\circ}$ attack on salicylic acid, 2,5-DHBA and 2,3-DHBA, although adequate was not optimal. Replacing Tris with phosphate buffer had a detrimental effect on this separation and also caused peak broadening. Therefore a different solvent system was devised using acetate buffer and methanol. Formulation of the gradient described in Materials and methods resulted in optimal separation of 2,5-DHBA (10.75 minutes) and 2,3-DHBA (13.72 minutes) as shown in fig 2A, and, with the use of diode array detection, a minor metabolite, namely catechol (8.98 minutes), was also detected (fig 2B) and can be 

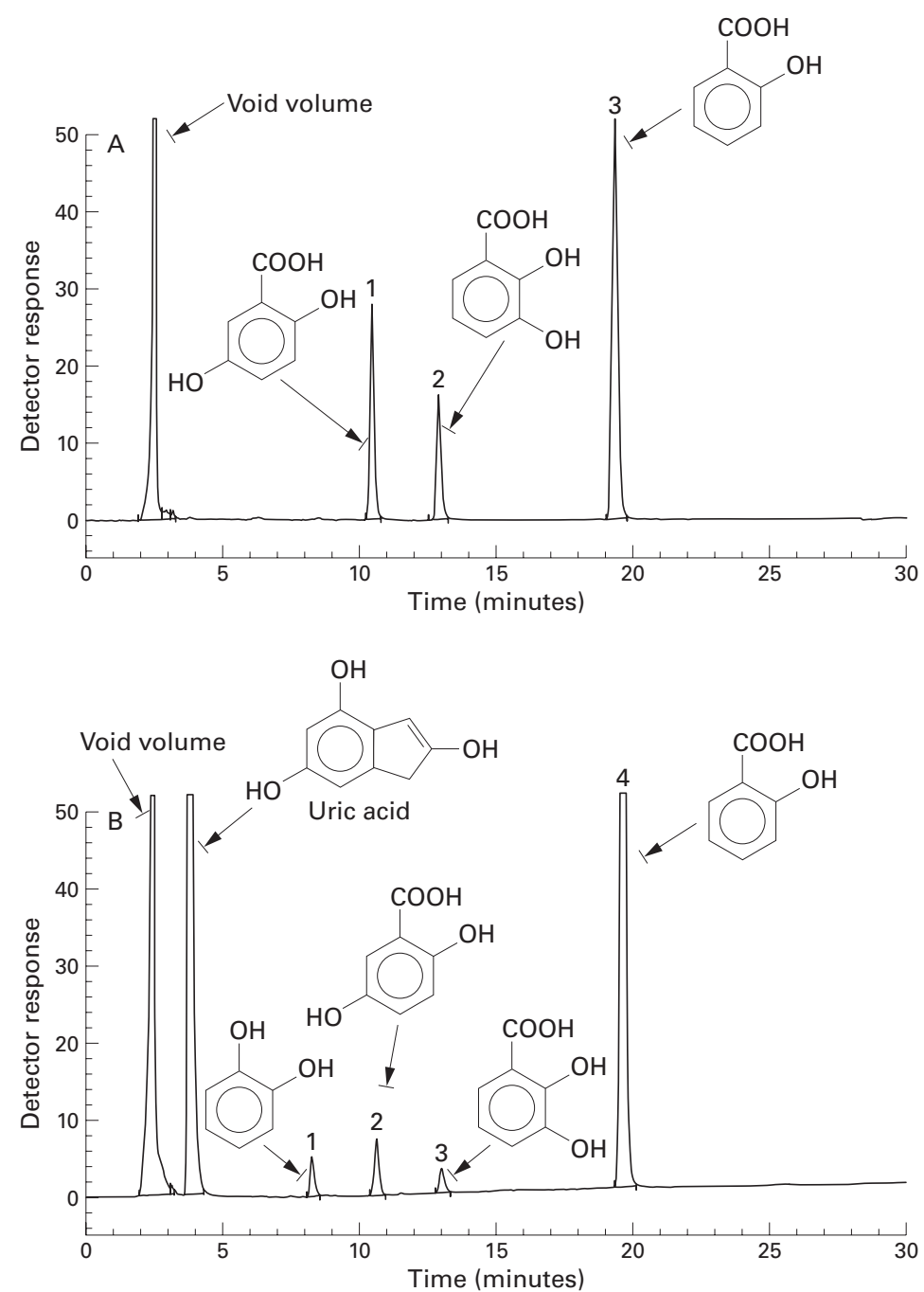

Figure 2 (A) High performance liquid chromatogram of salicylic acid (3) and its hydroxylated products 2,5-dihydroxybenzoic acid (1) and 2,3-dihydroxybenzoic acid (2) with diode array detector set at $325 \mathrm{~nm}$. (B) High performance liquid chromatogram of salicylic acid (4) and its decarboxylated product, catechol (1), and the hydroxylated products, 2,5-dihydroxybenzoic acid (2) and 2,3-dihydroxybenzoic acid (3), with the diode array detector set at $278 \mathrm{~nm}$ and $325 \mathrm{~nm}$.

quantified if deemed necessary. This HPLC method was used for all of the subsequent results reported here.

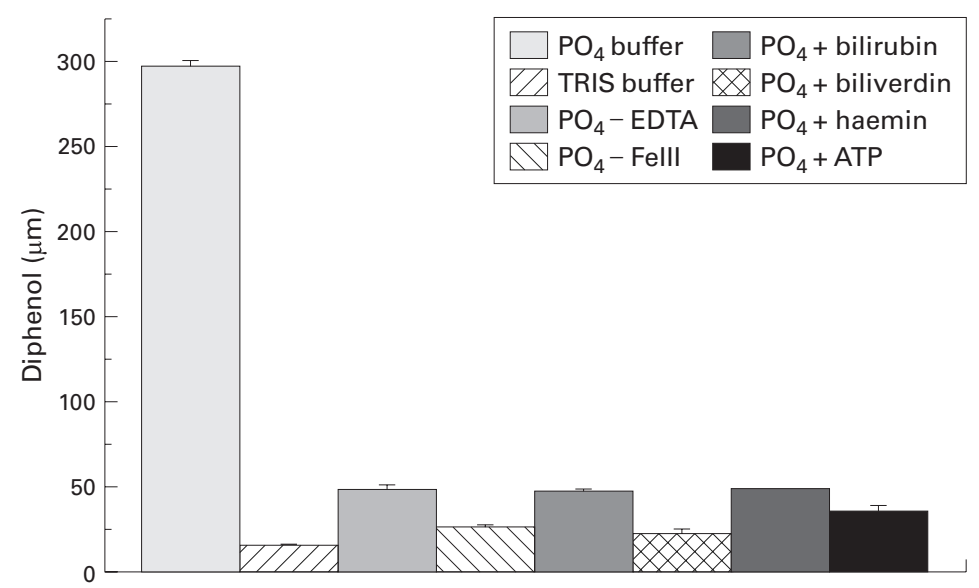

Figure 3 Comparison of the phosphate and Tris buffer systems for the detection of reactive oxygen species attack on salicylic acid.

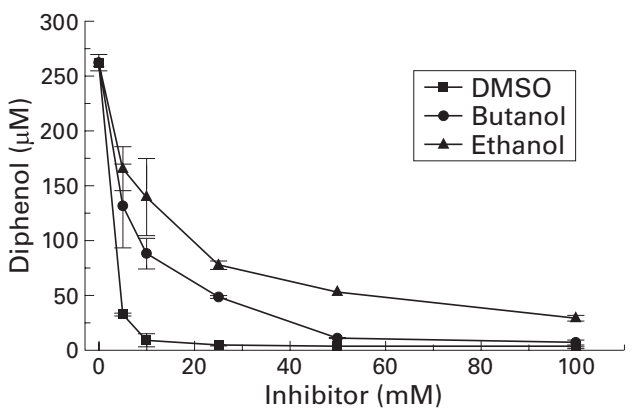

Figure 4 Effect of various scavengers on the detection of reactive oxygen species in the phosphate buffer system. DMSO, dimethyl sulphoxide.

COMPARISON OF THE TRIS AND PHOSPHATE METHODS IN THE HYPOXANTHINE/XANTHINE OXIDASE SYSTEM

A basic comparison of the two buffer systems used to support Fenton chemistry was conducted. Although many previous studies ${ }^{3} 12334$ using the hypoxanthine/xanthine oxidase system report the use of $\mathrm{pH} 7.4$, the optimum $\mathrm{pH}$ was found to be 6.5 in both the Tris and phosphate buffer systems. The data show (fig 3) that phosphate buffer at $\mathrm{pH} 6.5$ enables optimal detection of the products of $\mathrm{HO}^{\circ}$ attack on salicylic acid. The detection of these products (Tris buffer, $16.5 \mu \mathrm{M}$; phosphate buffer, $299 \mu \mathrm{M}$ ) was about 18 times greater in the phosphate system, showing that, as previously suggested, ${ }^{30}$ Tris is an efficient scavenger $(94 \%)$ of reactive oxygen species, the hydroxyl radical especially. Hydroxylation of salicylic acid was inhibited in a dose dependent manner (fig 4 ) by the classic scavengers of $\mathrm{HO}^{\circ}$, dimethyl sulphoxide $\left(\mathrm{IC}_{50}=1.77\right.$ $\mathrm{mM})$, butanol $\left(\mathrm{IC}_{50}=5.05 \mathrm{mM}\right)$, and ethanol $\left(\mathrm{IC}_{50}=10.17 \mathrm{mM}\right)$, and by the iron binding component (fig 5) of cereals, nuts, and pulses, phytic acid $\left(\mathrm{IC}_{50}=275 \mu \mathrm{M}\right)$. Further comparisons (fig 3) show that, as in the Tris system, generation of reactive oxygen species is severely curtailed on the omission of EDTA $(-83 \%)$ and iron $(-91 \%)$. Replacement of EDTA with other potential chelating agents such as bilirubin, biliverdin, and ATP as well as haemin (a source of redox active iron) were not shown to enhance free radical generation above that in the absence of EDTA.

Addition of ascorbic acid (fig 6), however, caused a dose dependent increase in the generation of reactive oxygen species either (at ascorbic acid concentration $500 \mu \mathrm{M}$ ) in the absence $(110 \%)$ or presence $(239 \%)$ of EDTA

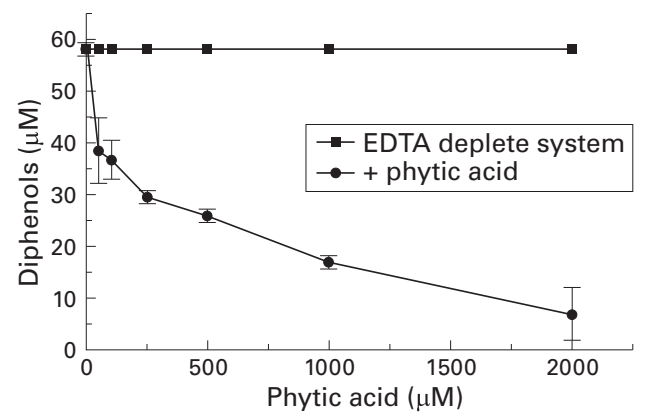

Figure 5 Inhibition of the generation of reactive oxygen species by phytic acid in an EDTA deplete phosphate buffer system. 


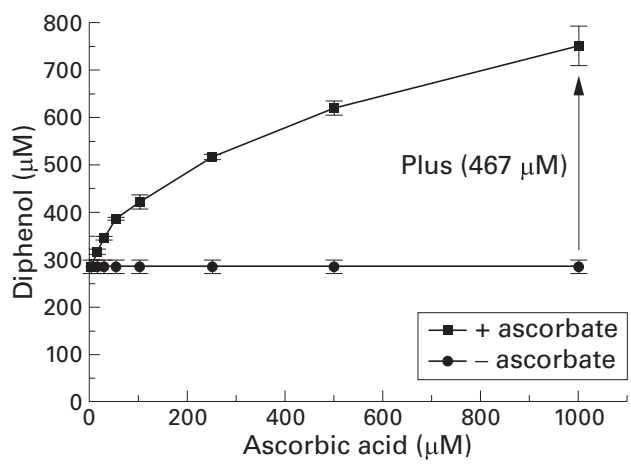

Figure 6 Promotion of the generation of reactive oxygen species by ascorbic acid in the complete phosphate buffer system.

(fig 7) consistent with other in vitro findings, ${ }^{31}{ }^{32}$ showing that the Udenfriend system comprising ascorbic acid, EDTA chelated iron, and hydrogen peroxide results in an approximate $15 \%$ conversion of the DNA base 2-deoxyguanosine to form the adduct 8 -hydroxy-2-deoxyguanosine. Ascorbic acid is a strong reducing agent, and here it probably enhances reduction of iron(III) to iron(II) by superoxide, allowing reaction with hydrogen peroxide which is also formed by the spontaneous dismutation of superoxide. These reactions appear to be enhanced when the iron is chelated, as judged by the substantial increase in dihydroxybenzoic acids produced in the presence of EDTA as opposed to in its absence.

INTERACTIVE EFFECTS OF EDTA, ASCORBIC ACID, AND PHYTATE ON GENERATION OF REACTIVE OXYGEN SPECIES

Because phytic acid, in an EDTA deplete hypoxanthine/xanthine oxidase system inhibits generation of reactive oxygen species in a dose dependent manner $\left(\mathrm{IC}_{50}=275 \mu \mathrm{M}\right)$ and given that addition of phytic acid (see below) to the faecal systems had no demonstrable effects on reactive oxygen species generation, it was of interest to elucidate whether chelators such as EDTA would interfere with the effects of phytate. The results (fig 8) of co-incubation experiments with phytic acid bound iron show that iron can be scavenged by the stronger chelator EDTA, at low concentrations especially. Scavenging of iron was maximal at an EDTA

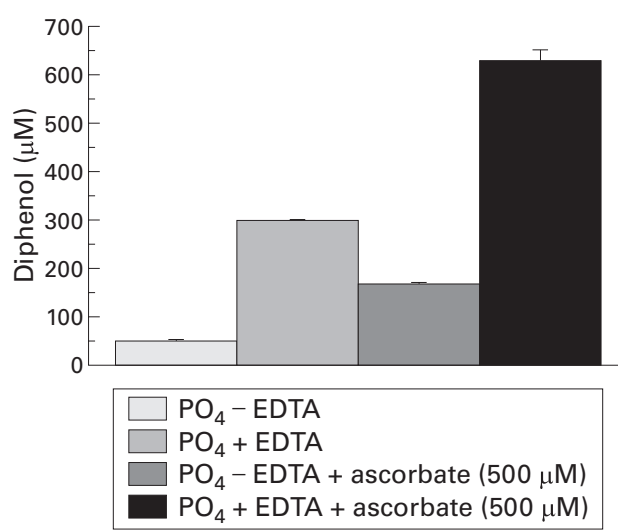

Figure 7 Effect of ascorbic acid $(500 \mu \mathrm{M})$ on the generation of reactive oxygen species in variations of the phosphate buffer system.

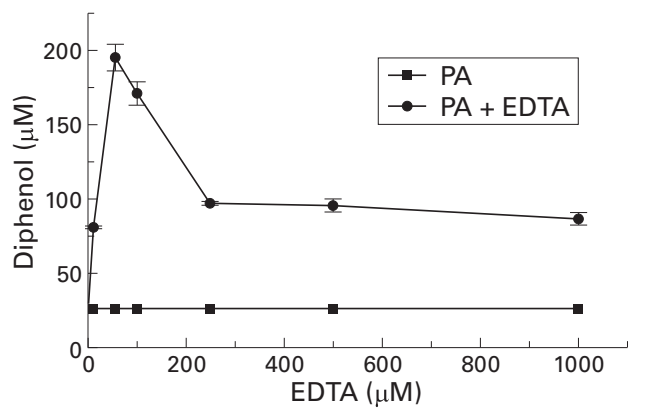

Figure 8 Effect of EDTA on phytic acid $(P A ; 500 \mu M)$ inhibition of generation of reactive oxygen species in the hypoxanthine/xanthine oxidase assay.

concentration of $50 \mu \mathrm{M}$ representing a ratio of phytate to EDTA of 10:1. At higher concentrations of EDTA, this effect, although significant, was less pronounced.

Although a chelator such as EDTA is not present in faeces, the data show that the phytate-iron complex can be compromised by the presence of competing species, which also affiliate with iron. To test this hypothesis further, experiments were conducted with ascorbic acid, which, although not a chelator of iron, is a powerful reducing agent, to assess its effect on phytate induced inhibition of free radical generation in the hypoxanthine/xanthine oxidase assay. Addition of ascorbic acid to phytate bound iron assays showed (fig 9) a significant dose dependent subversion of the inhibitory capacity of phytate on reactive oxygen species generation. At a concentration of ascorbic acid of $1 \mathrm{mM}$, the inhibitory effect was $100 \%$.

FAECAL GENERATION OF REACTIVE OXYGEN SPECIES In previous communications, ${ }^{2627}$ we have reported that the faecal matrix is capable of producing reactive oxygen species in abundance, as evinced from samples obtained during a calcium intervention trial. These data were generated using Tris buffer as the supporting medium, but the dynamics of the process were not studied. In view of this, and the fact that phosphate, as shown in this study, is superior to Tris buffer, we have examined and verified in detail the ability of the faecal matrix to support the generation of reactive oxygen species. For these studies, four samples were selected and subjected to a range of assays to confirm at least the involvement of free radicals in these processes.

Generation of reactive oxygen species in phosphate buffer by the faecal matrix, as for the

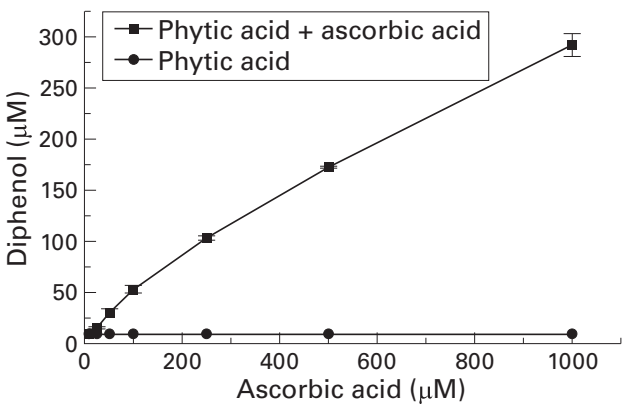

Figure 9 Effect of ascorbic acid on phytic acid (500 $\mu \mathrm{M})$ inhibition of reactive oxygen species generation in the hypoxanthine/xanthine oxidase assay. 


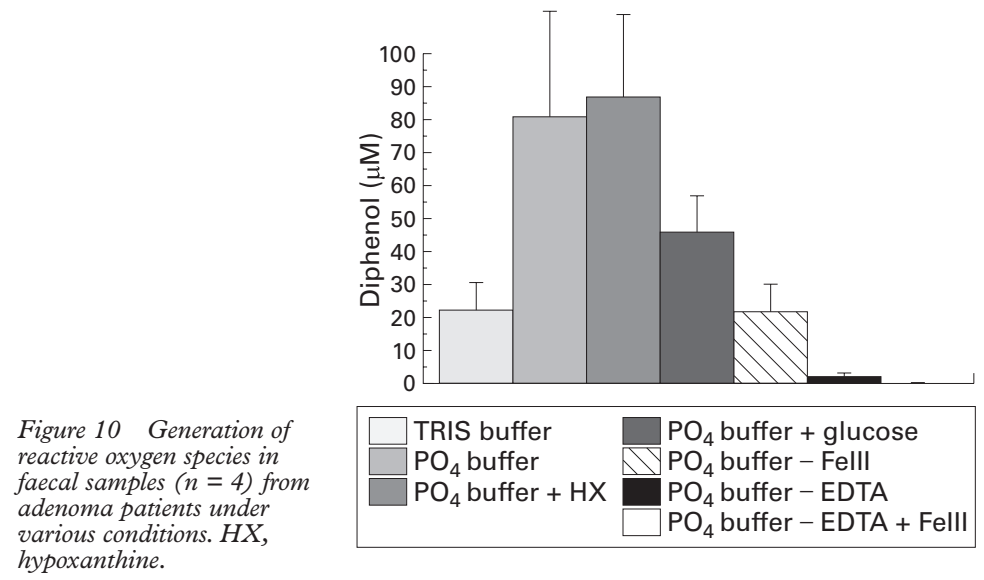

hypoxanthine.
Figure 11 Effect of various scavengers on the detection of reactive oxygen species in faecal samples ( $n$ = 4). DMSO, dimethyl sulphoxide. hypoxanthine/xanthine oxidase system, was far more efficient than in Tris buffer (fig 10). Hydroxylation of salicylic acid by the faecal sample incubates was inhibited in a dose dependent manner (fig 11) by the classic scavengers of $\mathrm{HO}^{\circ}$, dimethyl sulphoxide $\left(\mathrm{IC}_{50}=\right.$ $3.20 \mathrm{mM}$ ), butanol $\left(\mathrm{IC}_{50}=3.89 \mathrm{mM}\right)$, and ethanol $\left(\mathrm{IC}_{50}=8.31 \mathrm{mM}\right)$. This was also the case with catalase ( $\mathrm{IC}_{50}=13.2$ units), confirming the generation of $\mathrm{HO}^{\circ}$ by the faecal matrix.

Inclusion of hypoxanthine $(+7 \%)$ in the buffer (fig 10) had no significant effect on the generation of reactive oxygen species, showing that the samples did not contain viable xanthine oxidase, which obviously may have been a confounding factor. However, the sequential omission (fig 10$)$ of iron $(-75 \%)$, EDTA $(-97 \%)$, and iron and EDTA in combination $(-100 \%)$ caused significant attenuation of the HPLC signals and confirm findings in the hypoxanthine/xanthine oxidase model system. The different effects of iron depletion in the hypoxanthine/xanthine oxidase system $(-91 \%)$ and the faecal system $(-75 \%)$ probably reflect the ability of EDTA to scavenge iron from phytic acid in the faecal matrix, as shown in the hypoxanthine/xanthine oxidase assay. Addition of glucose to the phosphate buffer system, which was a component of the Tris buffer used by us ${ }^{26} 27$ previously, and by Babbs ${ }^{24}$ caused appreciable attenuation of the signals produced by the faecal matrix. This confirms previous reports ${ }^{35}$ that glucose, like other sugars, is a scavenger of free radicals.

When phytic acid was added to faecal samples in either complete phosphate buffer or EDTA deficient buffer, no significant change in reactive oxygen species generation occurred. These data are consistent with the hypoxanthine/xanthine oxidase system in the presence of EDTA, but are

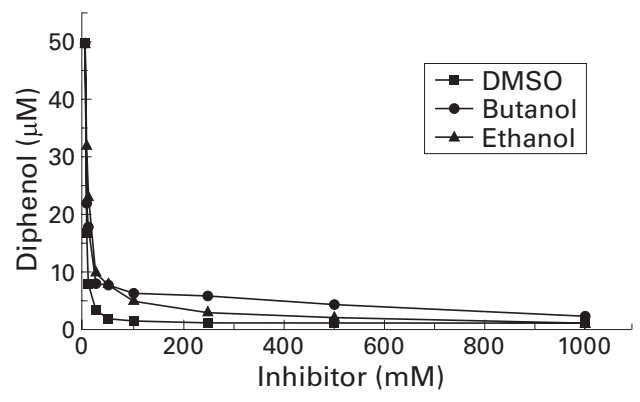

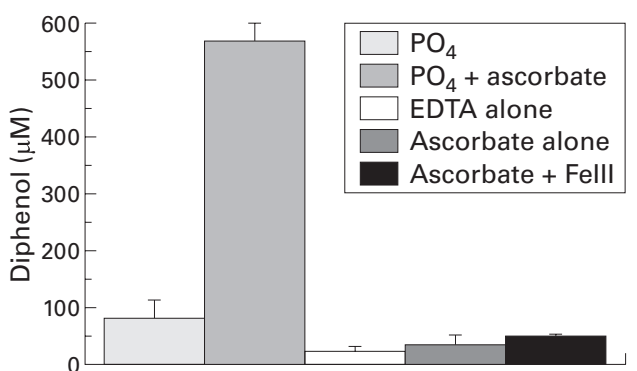

Figure 12 Effect of EDTA (500 $\mu \mathrm{M})$ and ascorbic acid $(500 \mu \mathrm{M})$ on the generation of reactive oxygen species in faecal samples.

at odds with observations in the absence of EDTA in which phytic acid causes a dose dependent decrease in the generation of reactive oxygen species. This is attributed to the strong iron chelating effect of phytate. Previous data ${ }^{23}$ showed a very strong correlation between faecal phytate and iron and on average a twofold excess of phytate, indicating that very little free iron, if any at all, exists in the faecal matrix. Addition of further phytate would therefore not be expected to reduce generation of reactive oxygen species.

Addition of ascorbic acid $(500 \mu \mathrm{M})$ to the faecal samples (fig 12) as in the hypoxanthine/ xanthine oxidase system had a similar promoting effect on the generation of reactive oxygen species. In the presence of EDTA (+598\%), the increase was about five times greater than in the hypoxanthine/xanthine oxidase assay. In the absence of EDTA, the increase was far less pronounced in both the absence $(+53 \%)$ and presence $(+121 \%)$ of iron. The reasons for these differences are not immediately clear.

\section{GENERATION OF REACTIVE OXYGEN SPECIES BY} FAECAL BACTERIA

$\mathrm{Babbs}^{24}$ attributed the generation of reactive oxygen species by faecal samples to the bacteria present, because, in his studies with autoclaved faeces, free radicals could not be detected. To clarify this situation, we cultured both the aerobic and anaerobic bacteria present in faeces in artificial media, and, after harvesting, examined a range of dilutions for their ability to produce reactive oxygen species. The results show conclusively that, in these samples, bacteria were not responsible for the generation of reactive oxygen species.

\section{GENERATION OF REACTIVE OXYGEN SPECIES IN} BACTERIAL FREE SYSTEMS

As the indigenous bacteria are not responsible for the generation of reactive oxygen species within the faecal matrix, what other component of the stool could be responsible? To attain some insights, we conducted experiments with filter sterilised and freeze dried faeces.

After addition to the complete buffer and thorough homogenisation, the faecal extracts were filter sterilised and incubated at $37^{\circ} \mathrm{C}$ for 18 hours. The results (table 1) show that, on average, the sterile samples were capable of supporting the generation of reactive oxygen species to an even greater extent than raw faeces. This enabled time course experiments (albeit at room temperature) to be conducted by HPLC for 
Table 1 Generation of reactive oxygen species by different faecal preparations

\begin{tabular}{lllll}
\hline Sample & $\begin{array}{l}2,5-D H B A \\
(\mu M)\end{array}$ & $\begin{array}{l}2,3-D H B A \\
(\mu M)\end{array}$ & $\begin{array}{l}\text { Catechol } \\
(\mu M)\end{array}$ & Total $(\mu M)$ \\
\hline $\mathrm{HX} / \mathrm{XO}$ & $122(0.7)^{\star}$ & $143(2.0)^{\star}$ & $35(0)^{\star}$ & $299(3)^{\star}$ \\
Faeces, wet $(100 \mathrm{mg})^{\star}$ & $39(14)$ & $47(16)$ & - & $87(30)$ \\
Faeces, wet $(100 \mathrm{mg}) \dagger$ & $9.6(3.5)$ & $12.9(4.9)$ & - & $22.4(8.4)$ \\
Faeces, wet $(100 \mathrm{mg}) \ddagger$ & $47(49)$ & $58(65)$ & - & $105(114)$ \\
Faeces, freeze dried $(25 \mathrm{mg}) \mathrm{S}$ & $27(9)$ & $34(15)$ & - & $61(24)$ \\
\hline
\end{tabular}

Experiments were conducted at $37^{\circ} \mathrm{C}$ as described in Materials and methods. Values are mean (SD) $\star$ Phosphate buffer, $\mathrm{pH}=6.50$.

$\dagger$ Tris buffer, $\mathrm{pH}=6.50$.

$\ddagger$ Phosphate buffer, $\mathrm{pH}=6.50$. Filter-sterilised as described in Materials and methods.

SPhosphate buffer, $\mathrm{pH}=6.50$. Representing on average the equivalent wet weight.

$\mathrm{HX} / \mathrm{XO}$, Hypoxanthine/xanthine oxidase; 2,5-DHBA, 2,5-dihydroxybenzoic acid; 2,3-DHBA, 2,3-dihydroxybenzoic acid.

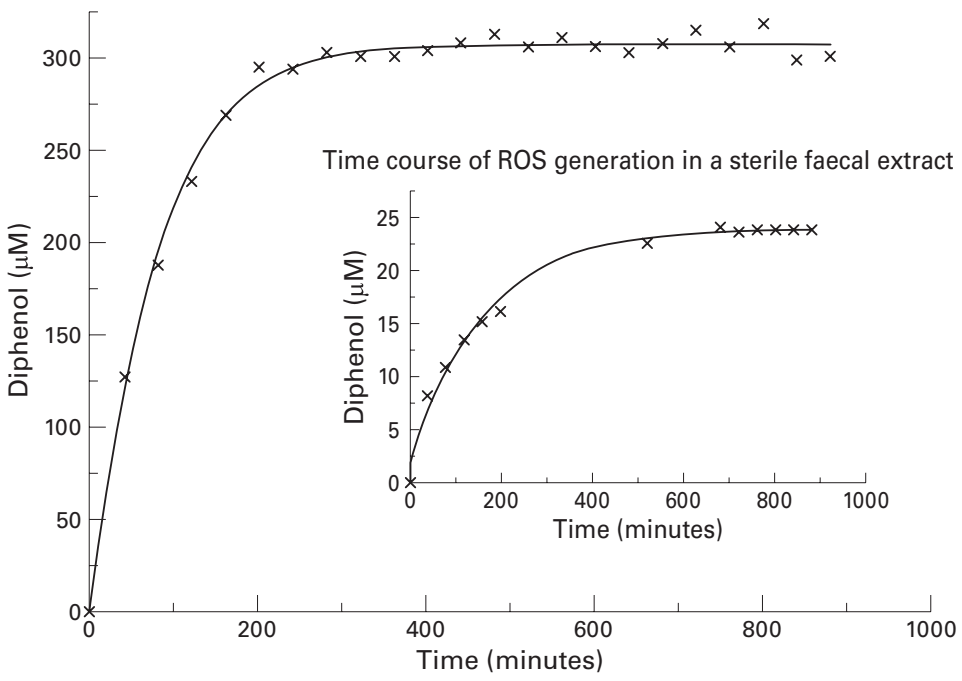

Figure 13 Time course of the generation of reactive oxygen species (ROS) in the complete phosphate hypoxanthine/xanthine oxidase system compared with that of faecal samples (inset). Note: The experiments were conducted at room temperature $\left(25^{\circ} \mathrm{C}\right)$ and the results are therefore not directly comparable with the data in table 1 (conducted at $37^{\circ} \mathrm{C}$ ).

comparison with the hypoxanthine/xanthine oxidase system. The curves for reactive oxygen species generation by both systems follow a similar cumulative pattern with time (fig 13). These data substantiated our results with faecal bacteria and further indicated that the ability of the faecal matrix to support Fenton chemistry cannot be attributed to the bacteria present. To support these observations, we compared faecal samples in both the wet and dry state. The freeze dried and wet faecal samples generated reactive oxygen species to a similar extent (table 1), confirming that the ability of the faecal matrix to support Fenton chemistry is due to a soluble factor.

\section{Discussion}

In this communication, we describe the refinement of an HPLC method ${ }^{26}{ }^{27}$ for the detection of reactive oxygen species generated in experimental systems. Substitution of Tris with phosphate buffer led to a substantial increase in the detection of free radicals, as measured using salicylic acid as an aromatic probe. Many previous studies ${ }^{31233}{ }^{34}$ have used $\mathrm{pH} 7.4$ as the optimum value for the hypoxanthine/xanthine oxidase system. In our studies, the $\mathrm{pH}$ optimum was found to be 6.5 , and this value was used for all the data reported.

Substitution of Tris with phosphate buffer afforded an 18-fold increase in the production and detection of hydroxylated products of salicylic acid in the hypoxanthine/xanthine oxidase system. With the faecal matrix, this was less pronounced in that only a fourfold increase was evident. These data, however, confirm that phosphate buffer is far more effective as a basal medium for the in vitro detection of reactive oxygen species. Why Tris buffer scavenges more reactive oxygen species in the hypoxanthine/ xanthine oxidase system is unclear.

Studies with the hypoxanthine/xanthine oxidase system using phosphate as the basal medium give clear signals in the HPLC for the two major hydroxylated products of salicylic acid, namely 2,5-DHBA (41\%) and 2,3DHBA (48\%), and the minor decarboxylated metabolite, catechol (11\%). These data are in excellent agreement with previous reports. ${ }^{75}$ Also the dynamics of the hypoxanthine/ xanthine oxidase system, although giving rise to far stronger signals, were identical in behaviour with those of the Tris buffer system. The increase in HPLC signals greatly enhanced the accuracy of the data generated for the detection of reactive oxygen species produced by faecal samples. In the Tris buffer system, it was necessary to extract the samples with diethyl ether and concentrate them 10-fold to detect the signals. Using phosphate buffer and the vastly improved HPLC method, free radicals generated by faecal samples can be analysed and quantified, after filtration, by direct injection on to the HPLC column. This not only decreases the analysis time but also greatly increases the precision of the method.

This has enabled elucidation of some of the dynamics of the system operating in the faecal matrix in which xanthine oxidase is simply replaced with raw, filter sterilised extracts, or freeze dried faeces. These data show that the ability of the faecal matrix to support Fenton chemistry in the complete phosphate system is influenced largely by the presence of EDTA chelated iron in the medium. The generation of reactive oxygen species in the hypoxanthine/ xanthine oxidase system is dependent (fig 1 ) on the formation of superoxide by xanthine oxidase as a byproduct of the hydroxylation of hypoxanthine via xanthine to uric acid. Superoxide so produced reduces the EDTA-Fe(III) chelate to EDTA-Fe(II), which readily reacts with hydrogen peroxide produced from the spontaneous dismutation of superoxide. The interaction of the EDTA-Fe(II) complex with hydrogen peroxide gives rise to $\mathrm{HO}^{\circ}$, as previously reported..$^{35}$ The $\mathrm{HO}^{\circ}$ radical in turn reacts with salicylic acid producing the hydroxylated products 2,5-DHBA and 2,3DHBA along with catechol. The mechanism of reactive oxygen species generation by the faecal matrix, however, is not exactly clear. Obviously the participation of xanthine oxidase can be ruled out because when hypoxanthine is included in the buffer it remains unmetabolised. Therefore the generation of reactive oxygen species by the faecal matrix operates through a different mechanism. It is possible that faeces contain a reducing agent somewhat similar to ascorbic acid, which reduces EDTA$\mathrm{Fe}$ (III) to EDTA-Fe(II), and the reduced complex reacts with hydrogen peroxide produced 
by the spontaneous dismutation of superoxide in the buffer. The presence in faecal systems of chelated iron is obviously as important as in the hypoxanthine/xanthine oxidase system because, in EDTA depleted buffer, the generation of reactive oxygen species is reduced by $97 \%$. In the absence of iron, this reduction was less pronounced $(-75 \%)$ and probably reflects the ability of EDTA to scavenge iron from phytic acid in the faecal matrix. In the absence of both iron and EDTA, the generation of reactive oxygen species could not be detected.

With regard to the faecal matrix, the following conclusions can be drawn. In the presence of suitably chelated iron the faecal matrix is capable of generating reactive oxygen species in abundance. However, in its absence, generation of reactive oxygen species is reduced by $97 \%$, indicating that, although factors that readily support Fenton chemistry exist in the matrix (in vitro), in the presence of suitably chelated iron it appears that, only if such a chelator exists in the intestine would oxidative stress be relevant in the large bowel. Furthermore, even if such conditions were to prevail, the generation of reactive oxygen species in the lumen must be regarded as innocuous because of the high reactivity of free radicals with other biomolecules. Nevertheless the generation of reactive oxygen species in or on the mucosal surface may be a result of a steep redox gradient where the reduced gut contents come into contact with the highly aerobic mucosa.

However, our data show, in contrast with previous beliefs, that reactive oxygen species are produced by a soluble factor within the faecal stream rather than by the indigenous bacteria. To date, the nature of this faecal component remains unknown but is likely to be a reducing agent. The soluble nature of the promoting factor renders it amenable to absorption, and circumstances may exist in which it comes into contact with either free or chelated iron in the colonocyte, leading to a direct attack on cellular DNA, or else initiates lipid peroxidation processes whereby membrane polyunsaturated fatty acids are attacked by $\mathrm{HO}^{\circ}$, propagating chain reactions leading to the generation of promutagenic lesions such as etheno based DNA adducts. ${ }^{10}$

Alternatively, generation of reactive oxygen species at the mucosal surface where the faecal water comes into contact with oxygen may cause damage to the protective mucus, leading to susceptibility to carcinogen exposure.

Studies are in progress to elucidate the nature of this soluble factor, and general screens in the framework of population and case-control studies are being carried out to establish whether or not Fenton chemistry can be supported by the faecal matrix in oxygen limiting atmospheres and whether these processes can be modulated by diet.

1 Halliwell B. Current status review: free radicals, reactive oxygen species and human disease: a critical evaluation with special reference to atherosclerosis. Br f Exp Pathol 1989;70:737-57.

2 McCord JM. Oxygen-derived radicals in post-ischemic tissue injury. N Engl f Med 1985;312:159-63.

3 Grootweld M, Halliwell B. Aromatic hydroxylation as a potential measure of hydroxyl radical formation in vivo: identification of hydroxylated derivatives of salicylate in human body fluids. Biochem ₹ 1986;237:499-504

4 Simmonds NJ, Allen RE, Stevens TRJ, et al. Chemiluminescence assay of mucosal reactive oxygen metabolites in inflammatory bowel disease. Gastroenterology 1992;103: 186-96.

5 Stich HF, Andrews F. The involvement of reactive oxygen species in oral cancers of betel-quid/tobacco chewers. Mutat Res 1989;214:47-61.

6 Nair UJ, Obe G, Friesen M, et al. Role of lime in the generation of reactive oxygen species from betel-quid ingredients. Environ Health Perspect 1992;98:203-5.

7 Keshavarzian A, Sedghi S, Kanofsky J, et al. Excessive production of reactive oxygen metabolites by inflamed production of reactive oxygen metabolites by inflamed ogy 1992;103:177-85.

8 Keshavarzian A, Olyaee M, Sontag S, et al. Increased levels of luminol-enhanced chemiluminescence by rectal mucosa of patients with colonic neoplasia: a possible marker for colonic neoplasisa. Nutr Cancer 1993;19:201-6.

9 Bartsch H, Nair J, Velic I. Etheno-DNA base adducts as tools in human cancer aetiology and chemoprevention. Eur 7 Cancer Prev 1997;6:529-34

10 Nair J, Vaca CE, Velic I, et al. High dietary omega-6 polyunsaturated fatty acids drastically increase the formation of etheno-DNA base adducts in white blood cells of female subjects. Cancer Epidemiology, Biomarkers and Prevention 1997;6:597-601.

11 Graf E, Eaton JW. Dietary suppression of colonic cancer. Fiber or phytate? Cancer 1985;56:717-18

12 Hill MJ, Giacosa A, Beckly D, et al. Consensus meeting on cereals, fibre and colorectal and breast cancers. Eur $\mathcal{F}$ Cancer Prev 1997;6:512-14.

13 Graf E, Mahoney JR, Bryant RG, et al. Iron-catalysed hydroxyl radical formation: stringent requirement for iron coordination site. $\mathcal{F}$ Biol Chem 1984;259:3620-4.

$14 \mathrm{Graf} \mathrm{E}$ (ed). Chemistry and applications of phytic acid: an overview. Minneapolis: Pilatus Press, 1986:1-21.

15 Graf E, Eaton JW. Antioxidant functions of phytic acid. Free Radic Biol Med 1990;8:61-9.

16 Shamsuddin AM, Elsayed AM, Ullah A. Suppression of large intestinal cancer in F344 rats by inositol hexaphosphate. Carcinogenesis 1988;9:577-80.

17 Shamsuddin AM, Ullah A. Inositol hexaphosphate inhibits large intestinal cancer in F344 rats 5 months after induction by azoxymethane. Carcinogenesis 1989;10:625-6.

18 Ullah A, Shamsuddin AM. Dose-dependent inhibition of large intestinal cancer by inositol hexaphosphate in F344 rats. Carcinogenesis 1990;11:2219-22.

19 Thompson LU, Zhang L. Phytic acid and minerals: effect on early markers of risk for mammary and colon carcinogenesis. Carcinogenesis 1991;11:2041-5.

20 Nelson RL, Yoo SJ, Tanure JC, et al. The effect of iron on experimental colorectal carcinogenesis. Anticancer Res experimental color

21 Siegers C-P, Bumann D, Baretton G, et al. Dietary iron enhances the tumor rate in dimethylhydrazine-induced colon carcinogenesis in mice. Cancer Lett 1988;41:251-6.

22 Siegers C-P, Bumann D, Trepkan HD, et al. Influence of dietary iron overload on cell proliferation and intestinal tumorigenesis in mice. Cancer Lett 1992;65:245-9.

23 Owen RW, Weisgerber UM, Spiegelhalder B, et al. Faecal phytic acid and its relation to other putative markers of risk for colorectal cancer. Gut 1996;38:591-7.

24 Babbs CF. Free radicals and the etiology of colon cancer. Free Radic Biol Med 1990;8:191-200.

25 Erhardt JG, Lim SS, Bode JC, et al. A diet rich in fat and poor in dietary fiber increases the in vitro formation of reactive oxygen species in human feces. $\mathcal{F}$ Nutr;127:706-9.

26 Owen RW, Wimonwatwatee T, Spiegelhalder B, et al. A high performance liquid chromatography system for quantification of hydroxyl radical formation by determination of dihydroxy benzoic acids. Eur f Cancer Prev 1996;5:233-40.

27 Owen RW, Spiegelhalder B, Bartsch, H. Phytate, reactive oxygen species and colorectal cancer. Eur $\mathcal{f}$ Cancer Prev 1998;7(suppl 2):S41-54.

28 Babbs CF, Gale MJ. Colorimetric assay for methanesulfinic acid in biological samples. Anal Biochem 1987;163:67-73.

29 Weisgerber UM, Boeing H, Owen RW, et al. Effect of longterm placebo-controlled calcium supplementation on sigmoidal cell proliferation in patients with sporadic adenomatous polyps. Gut 1996;38:396-402

30 Buxton GV, Greenstock CL, Helman WP, et al. Critical review of rate constants for reactions of hydrated electrons, hydrogen atoms and hydroxyl radicals in aqueous solution. Notre Dame: Radiation Chemistry Data Center, Radiation Laboratory, University of Notre Dame, 1986.

31 Kasai H, Nishimura S. Hydroxylation of deoxyguanosine at the C-8 position by ascorbic acid and other reducing agents. Nucleic Acids Res 1984;12:2137-45.

32 Floyd RA, Watson JJ, Wang PK, et al. Hydroxyl free radical adduct of deoxyguanosine: sensitive detection and mechanisms of formation. Free Radic Res Comms 1986;1:163-72.

33 Klein S, Cohen G, Cederbaum AI. Production of formaldehyde during metabolism of dimethyl sulfoxide by hydroxyl radical generating systems. Biochemistry 1981;20:6006-12.

34 Richmond R, Halliwell B, Chauhan J, et al. Superoxidedependent formation of hydroxyl radicals: detection of hydroxyl radicals by the hydroxylation of aromatic compounds. Anal Biochem 1981;118:328-35.

35 Halliwell B, Gutteridge JMC. Free radicals in biology and medicine. Oxford: Clarendon Press, 1993. 